\title{
DESCRIPCIÓN DE LA TERAPIA COGNITIVO-CONDUCTUAL PARA LA ANSIEDAD EN PACIENTES CON CÁNCER TERMINAL
}

\author{
DESCRIPTION OF THE COGNITIVE-BEHAVIORAL THERAPY FOR ANXIETY IN \\ PATIENTS WITH TERMINAL CANCER
}

Edgar Landa-Ramírez' Joseph Andrew Greer², Ariel Vite-Sierra³ ${ }^{3}$ Sofía Sánchez-Román ${ }^{4}$ y Angélica Riveros-Rosas ${ }^{5}$

I Facultad de Psicología/Programa de Medicina Conductual. Universidad Nacional Autónoma de México.

2 Department of Psychiatry Massachusetts General Hospital \& Harvard Medical School.

3 Facultad de Psicología, Universidad Nacional Autónoma de México.

${ }^{4}$ Instituto Nacional de Ciencias Médicas y Nutrición "Salvador Zubirán"

${ }^{5}$ Facultad de Contaduría y Administración, Universidad Nacional Autónoma de México

Resumen

El objetivo de este trabajo es describir los principales componentes y características de la terapia cognitivo conductual cuando se utiliza para tratar problemas de ansiedad en pacientes con cáncer terminal. A lo largo del escrito se describen los cuatro componentes que más se han utilizados en las investigaciones en este área: 1) principios de la terapia cognitivo conductual 2) técnicas de relajación 3 ) identificación y reestructuración de pensamientos negativos 4) planeación de actividades. De igual forma, se describe el promedio de duración de la terapia cognitivo conductual, número de sesiones, intervalos de aplicación y los principales problemas reportados cuando se aplica la terapia. Finalmente, se especifican recomendaciones para la aplicación de la terapia y para el desarrollo de líneas futuras de investigación en el área.

Palabras clave: Ansiedad, cáncer incurable, terapia cognitivo-conductual.

\section{Abstract}

The aim of this study was to describe the major components and features of cognitive behavioral therapy when used to treat anxiety in patients with terminal cancer. Specifically, four components of cognitive behavioral therapy for terminal cancer patients are described: 1) principles of cognitive behavioral therapy 2) relaxation techniques 3 ) identification and restructuring negative thoughts 4) planning activities. Similarly, the average duration of cognitive behavioral therapy, number of sessions, application intervals and major problems reported when therapy is applied are described. Finally, recommendations for the implementation of therapy and for the development of future research in this field are described.

Key words: Anxiety, incurable cancer, cognitive-behavioral therapy.

\section{Correspondencia:}

Universidad Nacional Autónoma de México.

Av. Universidad 31 14, Col. Copilco Universidad, Del. Coyoacán, 04510 México D.F., México,

E-mail: edgar_landa_ramirez@yahoo.com.mx

El presente trabajo forma parte de la investigación doctoral del primer autor bajo la dirección del último. 


\section{INTRODUCCIÓN}

El término de cáncer terminal, hace referencia a distintos tipos de cánceres, que debido a su avance no se pueden tratar de manera curativa, lo cual conlleva el progreso de la enfermedad hasta el faIlecimiento del paciente ${ }^{(1)}$. Los datos más recientes mencionan que de manera mundial al menos 7,6 millones de personas fallecen al año por algún tipo de cáncer y se calcula que en los próximos años, el cáncer va a disminuir en países desarrollados, pero se va a incrementar en países en vías de desarrollo ${ }^{(2-6)}$.

El paciente con cáncer terminal suele presentar una gran cantidad de síntomas físicos relacionados con el avance de la enfermedad, entre los más reportados se encuentran: fatiga, dolor, falta de energía, pérdida de apetito, anorexia, constipación, disnea $^{(7,8)}$. Razón por la cual, el tratamiento médico se enfoca primordialmente al control de los síntomas y al incremento -en la medida de lo posible- de la calidad de vida del paciente ${ }^{(1,9)}$. De igual forma, la evolución de la enfermedad puede traer una gran cantidad problemas emocionales, comportamentales, sociales y espirituales que afectan la calidad de vida del paciente y de las personas que lo rodean, por tal motivo, se busca apoyar de manera interdisciplinaria a los pacientes y cuidadores primarios durante la evolución de la enfermedad y continuar con los cuidadores posteriormente al fallecimiento del paciente ${ }^{(9,10)}$.

La ansiedad se ha informado como el problema emocional más común que enfrentan los pacientes con cáncer terminal, se ha llegado a calcular que se presenta en un rango del $7 \%$ al $48 \% \%^{(7,8,11,12)}$. Una posible explicación de que exista un rango tan amplio, es la forma en la cuál se conceptualiza y mide el problema, cuando se mide con el DSM-IV, dos estudios reportan $7,6 \%$ y $9,8 \%$ de ansiedad ${ }^{(11,12)}$. Pero cuando se llega a medir por medio de instrumentos de auto-reportes o preguntas directas se presentan porcentajes de $18 \%$ y $48 \%{ }^{(7,8)}$.

Estudios recientes correlacionan la ansiedad con una mayor presencia e intensidad de los síntomas físicos desagradables que experimentan los pacientes, se ha reportado, que se relaciona con la presencia de náusea, disnea y anorexia; y con una mayor intensidad de dolor, fatiga, pérdida de apetito, bienestar percibido, náusea, disnea, depresión, y anorexia ${ }^{(13,14)}$, estos datos incluso se han obtenido en pacientes hispanohablantes con cáncer terminal| ${ }^{(14)}$.

Una revisión reciente mencionó que se pueden utilizar medicamentos (benzodiazepinas e inhibidores selectivos de la recaptación de serotonina) junto con terapia psicológica (preferentemente terapia cognitivo conductual) para tratar la ansiedad en pacientes oncológicos en distintos estadios de la enfermedad ${ }^{(15)}$, sin embargo, cuando se buscó información proveniente de ensayos controlados aleatorizados en el área de cáncer terminal, los resultados fueron muy limitados para poder dar recomendaciones del uso de medicamento en esta población ${ }^{(15-17)}$.

En una revisión literaria realizada por los autores de este trabajo entre septiembre y diciembre de 2012 en los siguientes buscadores: Pubmed/Medline, Psyclnfo, Cochrane y Redalyc, se encontró que se han publicado doce estudios que han evaluado el efecto de la terapia cognitivo conductual (TCC) para el manejo de la ansiedad en pacientes con cáncer terminal con una expectativa de vida alrededor de seis meses ${ }^{(18-29)}$, sin embargo, solamente seis estudios utilizaron diseños experimentales, para evaluar el efecto de la terapia ${ }^{(22,25-29)}$. De los seis trabajos experimentales, en cinco se reportó que disminuyeron de manera estadísticamente significativa los puntajes de ansiedad entre la pre evaluación y post evaluación, lo cual parece indicar que la 
TCC es una muy buena opción para el manejo de ansiedad en esta población ${ }^{(25-29)}$.

Publicaciones recientes han señalado la importancia de dar detalles precisos del tratamiento no farmacológico, incluyendo el psicológico o comportamental, que se está impartiendo en una investigación, así como, de los componentes que pueden estar influyendo en el calculo del tamaño del efecto de la intervención, lo anterior con el objetivo de hacer más aplicable los datos de las investigaciones en ambientes reales ${ }^{(30-32)}$. No obstante lo anterior, se calcula que solo del $35 \%$ al $49 \%$ de las investigaciones no farmacológicas, reportan los detalles de sus intervenciones, y se comenta, que en las investigaciones comportamentales, éste ha sido el principal problema para poder crear guías clínicas ${ }^{(30)}$.

Aunque los estudios realizados de la TCC perecen indicar que es una buena opción para tratar a la ansiedad en los pacientes con cáncer terminal, pocas investigaciones realizaron una descripción precisa y a detalle del tratamiento que emplearon, lo cual hace que la intervención empleada se convierta en una "caja negra" que puede comprometer la validez externa de los hallazgos de la intervención ${ }^{(31)}$. Por esta razón, se considera pertinente realizar una descripción de la TCC y de los componentes que se emplean cuando se aplica dicha terapia, es así, que el objetivo de este escrito es describir los principales componentes que se utilizan en la TCC para el abordaje de la ansiedad en pacientes con cáncer terminal. En este estudio se recurrió a la descripción de los componentes empleados en las doces investigaciones previamente mencionadas que han evaluado el efecto de la terapia.

\section{Componentes de la TCC}

A continuación se describirán los cuatro componentes más utilizados en la TCC para la ansiedad en pacientes con cáncer terminal así como, las principales características de las sesiones en las que se imparten.

\section{Principios de la terapia cognitivo conductual}

De manera general se busca que los pacientes identifiquen la relación que existe entre sus pensamientos, comportamientos, emociones y síntomas físicos. Se recomienda comenzar preguntando al paciente de una manera abierta su experiencia con el cáncer, su tratamiento, el tiempo que lleva con su diagnóstico y preocupaciones sobre su enfermedad ${ }^{(20)}$. Se debe de ser muy cuidadoso ya que se ha reportado que hasta un $20 \%$ de los pacientes que reciben por primera vez a cuidados paliativos, no saben sobre su diagnóstico de cáncer terminal(14).

Como parte de la evaluación, el terapeuta debe tratar de identificar, pensamientos, comportamiento y emociones que puedan estar relacionados con la presencia de ansiedad. El terapeuta deberá de ser muy claro al explicarle al paciente, que la ansiedad tiene componentes cognitivos, comportamentales y emocionales y por tal razón, es necesario identificar cuáles de estos se encuentran presentes en el paciente para poder ayudarlo a disminuirlo.

Los componentes cognitivos son muy importantes para identificar y modificar pensamientos irracionales, irreales, reales o negativos que están asociadas a la ansiedad de los pacientes ${ }^{(33)}$. Se recomienda ampliamente, comenzar utilizando situaciones de la vida diaria del paciente, para ejemplificar la relación entre pensamientos, emociones, síntomas físicos y comportamientos; posteriormente, se podrá dar énfasis en cómo los pensamientos negativos con respecto a la enfermedad pueden llevar a los pacientes a sentirse ansiosos y eso lo llevará a realizar cierto tipo de comportamientos y experimentar una gran cantidad 
de síntomas físicos ${ }^{(19)}$. Un estudio utilizó el modelo de Padesky (véase figura 1) para enfatizar de manera ilustrada la relación entre los pensamientos, el estado de ánimo, las conductas, las reacciones físicas ${ }^{(19)}$.

Los componentes comportamentales han sido considerados como parte indispensable en el tratamiento de la ansiedad, se debe de hacer énfasis al paciente, que este componente hace referencia a dos grandes áreas: 1) incrementar conocimientos, habilidades y actividades con respecto a sus capacidades actuales. 2) disminuir la evitación de actividades, situaciones o comportamientos problemáticos ${ }^{(33,34)}$. En el caso del cáncer avanzado, la evitación se puede presentar en la falta de adherencia al tratamiento paliativo del paciente, en un incremento excesivo en el monitoreo de síntomas físicos, o varios de los pacientes pueden perder motivación e interés en realizar actividades por ejemplo, las sociales ${ }^{(20,34)}$. En caso de que el paciente, muestre un marcado deterioro en la realización de actividades, se puede utilizar un registro de actividades programadas semanalmente; dicho registro se puede llenar de manera conjunta entre el paciente y terapeuta, y se deberá de tener mucho cuidado de programar actividades que se correspondan de manera real con las capacidades físicas del paciente y no deberá de perderse de vista el programar actividades que sean placenteras para el paciente reforzando así, la realización de las mismas.

Finalmente, se recomienda dar mucho énfasis en los componentes emocionales y los síntomas físicos, se deberá de describir al paciente, cómo las distintas emociones, por ejemplo la tristeza, ansiedad, desesperación se relacionan con la realización de comportamientos de evitación o con que las personas tengan algún pensamiento negativo en particular. De igual forma, se recomienda ser muy enfático en el hecho de que varios síntomas físicos que experimentan los pacientes se exacerban cuando presentan estados de ánimo ansioso y deprimido. $Y$ se dará énfasis en que una forma de ayudar a controlar la presencia de los síntomas desagradables, se puede dar por medio de ejercicios enfocados a romper ciclos en los cuales estén presentes

Figura 1. Modelo de Padesky

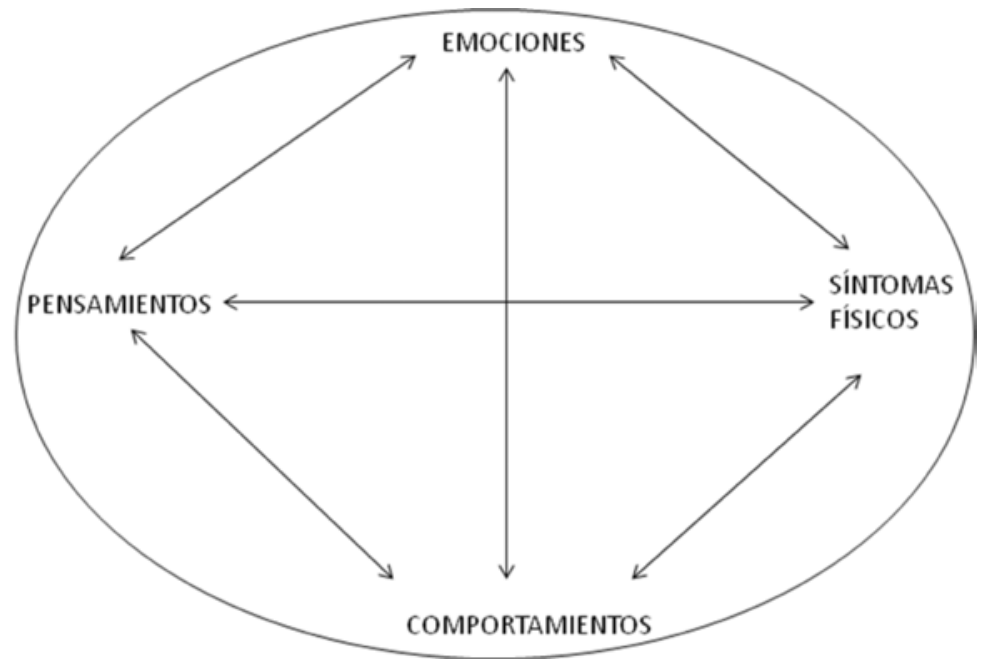


estados de ánimo como la ansiedad y los pensamientos y comportamientos asociados: por ejemplo: cuando estoy muy angustiado (componente emocional) prefiero no salir de mi casa y evito hacer cosas que me gustan; como ver mi programa de televisión favorito (evitación comportamental) y ahí es cuando suelo pensar que mi enfermedad está avanzando (componente cognitivo) y siento menor control del dolor (componente físico).

\section{Técnicas de relajación}

Las técnicas de relajación fueron utilizadas en varios estudios, las más empleadas fueron: relajación muscular progresiva, relajación autógena, imaginación guiada y respiración de labios fruncidos ${ }^{(20,24,28,29)}$.

Algunos autores han recomendado que antes de comenzar con la aplicación de la técnica de relajación, se enseñe al paciente de manera breve y concisa la respuesta de "pelea y huída" y los cambios fisiológicos producidos por el sistema nervioso simpático cuando se detectan señales de amenaza, por ejemplo: respiración rápida, palpitaciones cardíacas elevadas, entumecimiento, sudoración, cambios visuales ${ }^{(20,29)}$.

Se recomienda decirle al paciente que independientemente del origen de los síntomas (por el avance de la enfermedad, por la ansiedad en sí misma, por los medicamentos o la combinación de las tres) la relajación podrá ser de ayuda para controlar la ansiedad y se recomienda dar énfasis en la importancia de la practica y repetición, para obtener mejores resultados ${ }^{(20)}$.

Debido a que varios de los pacientes con cáncer terminal experimentan diversos síntomas físicos como dolor o fatiga se ha recomendado utilizar técnicas de relajación que no se interpongan con dichos síntomas (relajación autógena o imaginación guiada ${ }^{(20,24)}$. En caso de que el paciente no presente exacerbación de síntomas físicos, se podrá utilizar la relajación muscular progresiva ${ }^{(24,28)}$.

Si el paciente presenta un deterioro cognitivo importante, no se recomienda utilizar la relajación autógena o imaginación guiada, ya que no existe la reactividad comportamental básica para seguir instrucciones. Finalmente, si el paciente presente disnea se ha recomendado ampliamente el uso de la respiración de labios fruncidos, ya que es una técnica muy empleada para el control de este problema en paciente con enfermedad pulmonar obstructiva crónica ${ }^{(35)}$. y de manera reciente se ha utilizado en pacientes con disnea debida al cáncer pulmonar avanzado $^{(20)}$.

Finalmente, en varios de los estudios se utilizaron audiograbaciones para exponer al paciente a las mismas condiciones de relajación; además de hacerle más factible que utilizara la misma cuando le pareciera conveniente $\mathrm{e}^{(20,23,24,28,29)}$.

\section{Identificación y reestructuración de pensamientos negativos}

Fue una de las técnicas más utilizadas en las investigaciones consultadas ${ }^{(18-21,23,25-27,29)}$, se empleó de manera muy similar a la forma en la que se aplica con poblaciones sin enfermedad aparente ${ }^{(33}$, sin embargo, fue modificada y adaptada a las necesidades particulares de los pacientes terminales. La principal modificación que se realizó en la reestructuración fue la forma en la que se clasifica y abordan los pensamientos de los pacientes; ya que en población general se suele trabajar con pensamientos disfuncionales o irreales, mientras que en los pacientes con cáncer terminal, se podría trabajar además, con pensamientos que pueden ser reales, racionales pero disruptivos (por ejemplo voy a morir de cáncer y con mucho dolor; me duele mucho más que antes; me preocupa que cada vez respiro con más dificultad) 
por lo cual es indispensable evaluar y tomar decisiones con respecto a cómo tratar estos pensamientos. Se recomienda que en caso de que el pensamiento sea irreal, se identifiquen los errores cognitivos y se utilicen técnicas convencionales de la TCC para abordarlos desarrollando respuestas cognitivas racionales ${ }^{(20,29)}$.

En caso de que el pensamiento del pacientes sea real, se recomienda preguntarse si hay algo que se pueda hacer; si la respuesta es afirmativa, se recomienda enseñarle al paciente a aplicar los componentes de solución de problemas, la cual ha sido investigada y adaptada para pacientes con cáncer y cuidadores de pacientes paliativos ${ }^{(36-38)}$

En caso de que el paciente tenga un pensamiento real y no pueda hacer nada para modificar el problema, se recomienda utilizar los principios de la terapia de aceptación; en la cual se le pide al paciente que identifique y se exponga a los pensamientos que le provocan ansiedad, pero de una manera paulatina, abierta, flexible y evitando los juicios de valor, como si fuera un observador externo, para posteriormente, aumentar su tolerancia a la situación y poder realizar acciones que le permitan estar más tranquilo ${ }^{(39)}$.

De igual forma, se ha reportado que varios de los de los pacientes con cáncer terminal, tienen pensamientos que son reales en cuanto al deterioro de su salud y estos pueden ser disruptivos y generadores de ansiedad por lo cual, se propone identificar qué pensamientos negativos están desarrollando y una vez identificados, se deberá de ayudar a los pacientes a modificar los mismos. Lévesque et al. han ejemplificado algunos de los pensamientos que tienen pacientes con cáncer de mama terminal(26).

Si no es claro para el psicólogo si el pensamiento que está evaluando tiene bases reales o irreales, se recomienda acudir con el personal de salud para poder tomar la decisión al respecto (véase algo- ritmo para el cáncer avanzado figura 2$)^{(20)}$. Finalmente, se recomiendan pedir a los pacientes o cuidadores primarios informales que lleven registros de los pensamientos que los están molestando, buscando siempre que estos contengan los siguientes criterios: sean breves; se distingan entre pensamientos, situaciones y emociones; escriban oraciones y no preguntas; se escriban separados; y realmente contengan pensamientos $^{(34)}$.

\section{Planificación de actividades}

En la mitad de los estudios revisados, se consideró indispensable trabajar en la planeación de actividades generales, metas y tareas diarias (limpiar la casa, cocinar, trabajar, jugar golf) las cuales fueran acordes

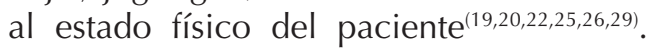
Uno de los principales problemas identificados en los estudios, fue que debido al avance de la enfermedad, los pacientes estaban muy fatigados lo cual influyó en que dejaran de hacer las actividades en su día a día, fomentando así, el desarrollo de ansiedad y depresión ${ }^{(20)}$.

Debido a lo anterior, en un par de investigaciones se trabajó con los pacientes para que identificaran el nivel de energía que tenían cada día; y con base en dicho nivel de energía, pudieran planear el tipo de actividades que realizaban durante ese día ${ }^{(20,29)}$. Lo anterior ayudó a que el paciente se sintiera más satisfecho, ya que sus expectativas de actividades estaban acordes a su nivel físico. De igual forma, el paciente aprendía a prescindir de las actividades que le requerían mucho esfuerzo y que probablemente no podía realizar (cocinar una comida completa) sustituyéndolas por actividades que eran más probables que sí pudiera conseguir (ayudar a cocinar picando alimentos).

Greer et al. ${ }^{(20,29)}$ han propuesto que tanto el terapeuta como el paciente, de manera conjunta realicen categorías que corres- 


\section{Tabla 1. Cambio de pensamientos negativos}

\section{Pensamientos en cáncer terminal}

Pensamientos negativos

"Voy a morir sola y con dolor"

"No tiene sentido seguir viviendo, cuando ya sé que voy a fallecer"

"Ya no soy útil para mi familia, solo soy una carga"

"Toda esta situación me está sobrepasando ya no puedo aguantarla".
Pensamientos positivos

"Sé que la gente más importante para mí, va a estar ahí cuando yo muera y mi doctor me ha asegurado que tendré suficiente medicamento para controlar el dolor"

"Nadie sabe el tiempo que va a vivir y todavía puedo tener suficiente tiempo para hacer actividades agradables con mi familia y alcanzar algunas metas que son importantes para mí"

"Es verdad que ya no puedo hacer muchas de las cosas que antes hacía pero estoy seguro de que mi familia está feliz de tenerme cerca y de cuidar de mí"

"Aunque la situación es muy complicada, puedo aprender nuevas habilidades que me ayuden a enfrentarla"

Figura 2. Algoritmo de TCC en cáncer avanzado propuesto por Greer et al. $2010^{(20,40)}$.

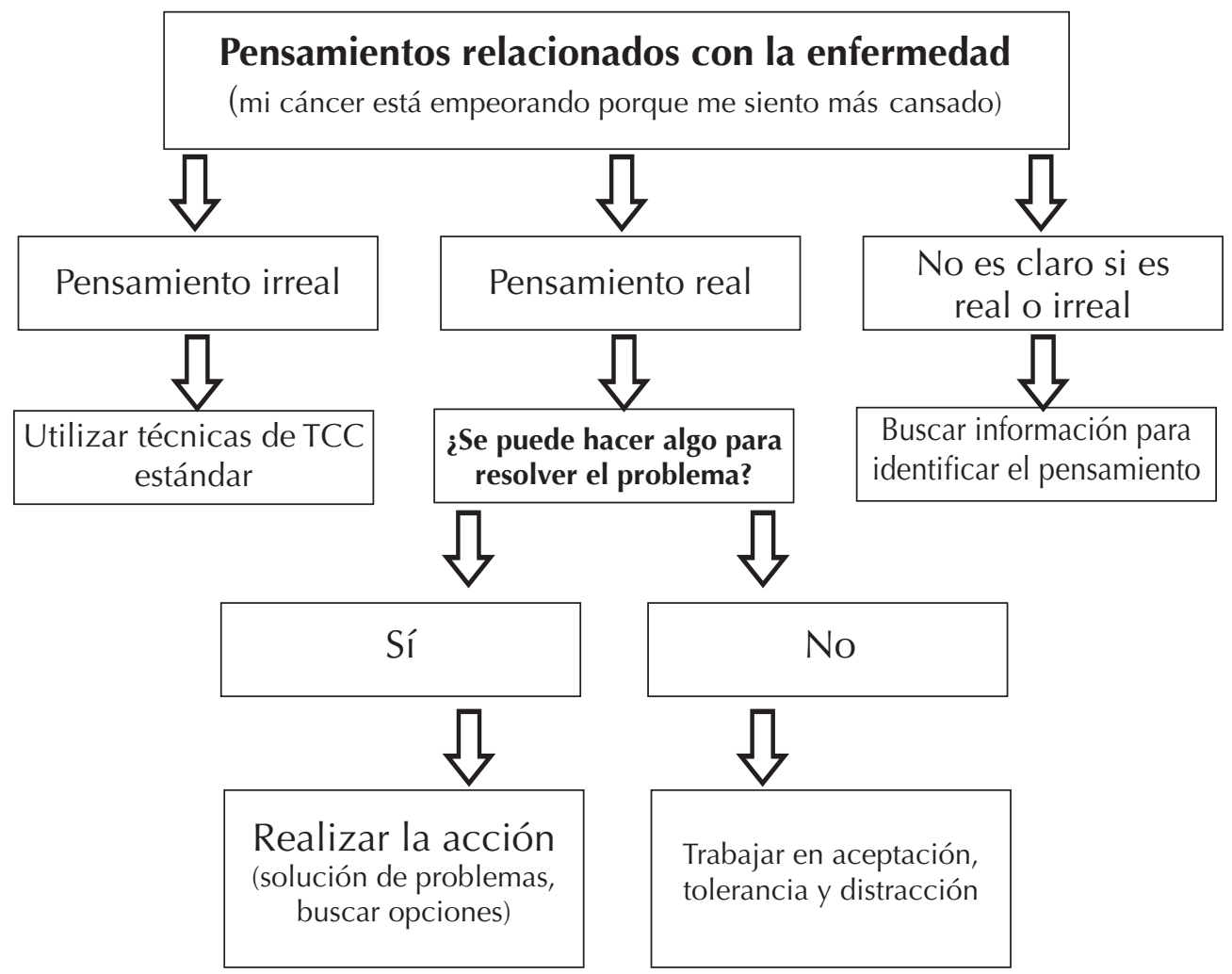


pondan a la fuerza que tiene el paciente para realizar actividades; se nombraba "día A" cuando el paciente tenía energía suficiente para realizar del $75-100 \%$ de sus actividades diarias. Se nombraba "día B" cuando el paciente tenía energía para realizar del $50-75 \%$ de sus actividades. Finalmente, en el "día C" cuando el paciente solo tenía energía para realizar menos del $50 \%$ de actividades durante ese día.

De igual manera, se consideró muy importante llevar a cabo la planeación de actividades placenteras que estuvieran acordes con el desarrollo de la enfermedad de cada paciente ${ }^{(20,25,26,29)}$, en ocasiones se utilizó la modalidad en la cual los pacientes dejaran de hacer actividades que los hicieran sentir cansados (como limpiar la casa) para dar preferencia a actividades lúdicas o de descanso ${ }^{(25,26)}$. Finalmente, en cuatro estudios se trabajó para fomentar que el paciente estableciera metas reales a corto y mediano plazo ${ }^{(25,26)}$, además de que anticipara acciones en caso de que sus síntomas físicos empeoraran ${ }^{(21,28)}$.

Finalmente, hubo otra variedad de técnicas que se utilizaron con los pacientes de cáncer terminal algunas de ellas fueron: técnicas de solución de problemas, manejo de estrés e ira, técnicas de meditación, comunicación asertiva, auto estima y aceptación, relaciones familiares y formulación de preguntas para el personal de salud.

\section{Características generales de la TCC}

De los doce estudios revisados, ocho trabajos emplearon terapia individual y cuatro utilizaron terapia grupal; a pesar de que esta última reportó efectos benéficos para los pacientes ${ }^{(18,21,23,25)}$ se ha llegado a reportar que no permite atender plenamente las necesidades individuales de los pacientes con cáncer terminal(26), además de que aumenta el riesgo de que haya pro- blemas emocionales en los pacientes que asisten a los grupos cuando se presenta el fallecimiento de sus compañeros ${ }^{(23)}$, por lo cual, la terapia individual podría tener más beneficios para los pacientes.

Las terapias se impartieron en promedio en 6 sesiones, (en un rango de 2 a 11 sesiones). El promedio de duración de cada sesión fue de 80 minutos, con un intervalo que iba de 15 a 120 minutos. Las terapias se trataban de dar de manera semanal, pero hubo varios estudios que reportaron que debido al avance de la enfermedad se le dificultaba mucho a los pacientes asistir a la terapia o lo hacían en condiciones poco óptimas para recibirla ${ }^{(19,23,27)}$. Atendiendo lo anterior, algunos estudios están desarrollando modalidades distintas para aplicar la TCC, por lo cual se han comenzado a dar en el hogar del paciente ${ }^{(27)} \mathrm{O}$ incluso por videoconferencia ${ }^{(41,42)}$.

\section{CONSIDERACIONES FINALES}

A pesar de que la TCC es una opción benéfica para el abordaje de la ansiedad en pacientes con cáncer terminal, aún quedan varios problemas pendientes por abordar en el área. Un problema es cómo aplicar la terapia, se recomienda que el personal de salud, sea muy sensible y cuidadoso cuando aplique los componentes de la TCC previamente descritos; es ampliamente recomendable seguir los pasos de la práctica basada en el evidencia para tomar la decisión clínica de qué tratamiento aplicar y cómo hacerlo con cada uno de los pacientes ${ }^{(43-45)}$. Se deberá de integrar a la evidencia empírica de la terapia, los deseos y expectativas que tienen los pacientes con respecto de la misma ${ }^{(46)}$, de igual forma, se tendrá que identificar el contexto institucional en el que se encuentra el paciente para decidir si es pertinente aplicar la TCC o buscar un tratamiento distinto pero con evidencia acorde a las necesidades del pacientes ${ }^{(15,47)}$. El personal 
de salud, deberá de ser flexible para aplicar el componente indicado a las necesidades del paciente con cáncer terminal, es así, que si se tenía contemplado trabajar en la reestructuración de pensamientos negativos asociados al cáncer, pero se observa que el paciente en ese momento puede beneficiarse más con la aplicación de técnicas de relajación, el personal de salud, no deberá de dudar en aplicar el segundo componente, para el beneficio del paciente. De igual forma, dado el deterioro constante del paciente, se recomienda involucrar a los cuidadores primarios informales, para que ellos también aprendan las habilidades enseñadas en la TCC y que a su vez, promuevan en el paciente el uso de las mismas.

Otro punto importante, es la necesidad imperante de generar una mayor cantidad de investigaciones que aporten datos empíricos acerca del efecto de la TCC en esta población, sin embargo, el generar este tipo de investigaciones es sin duda un reto importante, ya que la expectativa de vida tan corta de los pacientes y el marcado deterioro en ellos, ha generado que incluso únicamente se puedan reclutar alrededor del $10 \%$ total de pacientes para las investigaciones $^{(25,27)}$ y que a las seis semanas de la investigación, se tenga hasta un $43 \%$ de pacientes perdidos ${ }^{(27)}$. Aunque el ensayo controlado aleatorizado, sea el "gold standard" en la investigación actual, el tener muertes experimentales arriba del $40 \%$ representa una fuerte amenaza a la aleatorización empleada y es una fuente de sesgos a la validez interna de la investigación ${ }^{(48)}$. Lo anterior cuestiona ampliamente el uso de ensayos controlados aleatorizados en pacientes con cáncer terminal, lo cual abre la puerta al uso de otros diseños experimentales como el de $\mathrm{N}=1^{(49)}$, el cual ya ha sido evaluado en pacientes con cáncer de mama terminal(26).

Finalmente, es factible mencionar que existe una gran área de oportunidad para realizar investigaciones de la TCC en pacientes con cáncer terminal; algunas futuras líneas de investigación podrían incluir trabajos que repliquen efectos de la TCC en ansiedad en poblaciones con características muy particulares, como bajo nivel económico, educativo, o con problemas de memoria asociada a la enfermedad. De igual forma, se vuelve relevante evaluar el efecto de nuevas modalidades de TCC (en el hogar, videoconferencia, telefónica) y sus efectos en variables emocionales. También, se puede trabajar para evaluar el efecto de la TCC en pacientes con ansiedad grave, ya que la mayoría de los trabajos solo la han evaluado en población con ansiedad leve y moderada. Una área que podría ser vital en la investigación psicológica futura, será la propuesta de diseños de investigación que sean más adecuados a las necesidades y características tan particulares de la población terminal. Finalmente, se puede trabajar en estudios que evalúen el efecto de TCC en pacientes con cáncer muy avanzado cuya expectativa de vida sea menor a tres meses, condición que padecen una gran cantidad de pacientes con cáncer terminal en países en vías de desarrollo ${ }^{(14,38)}$.

\section{REFERENCIAS BIBLIOGRÁFICAS}

1. World Health Organization. Palliative care: cancer control knowledge into action En: WHO, editor. Guide for effective programs. [Acceso 1 de marzo de 2010]. Disponible en: http://whqlibdoc.who.int/publications/2007/9241547345_eng.pdf?ua=1

2. Jemal A, Bray F, Center MM, Ferlay J, Ward E, Forman D. Global cancer statistics. CA Cancer J Clin 2011;61:69-90. Doi: 10.3322/caac.20107.

3. Jemal A, Center MM, DeSantis C, Ward EM. Global patterns of cancer incidence and mortality rates and trends. Cancer Epidemiol Biomarkers Prev 2010;19:1893-907. Doi: 10.1158/1055-9965.EPI-10-0437. 
4. Bray F, Jemal A, Grey N, Ferlay J, Forman D. Global cancer transitions according to the Human Development Index (20082030): A population-based study. Lancet Oncol 2012;13:790-801. Doi: 10.1016/ S1470-2045(12)70211-5.

5. Siegel R, Ma J, Zou Z, Jemal A. Cancer statistics, 2014. CA Cancer J Clin 2014;64:929. Doi: 10.3322/caac.21208.

6. Goss PE, Lee BL, Badovinac-Crnjevic T, Strasser-Weippl K, Chavarri-Guerra Y, St Louis J, et al. Planning cancer control in Latin America and the Caribbean. Lancet Oncol 2013;14:391-436. Doi: 10.1016/ S1470-2045(13)70048-2.

7. Laugsand EA, Kaasa S, de Conno F, Hanks G, Klepstad P; Research Steering Committee of the EAPC. Intensity and treatment of symptoms in 3,030 palliative care patients: a cross-sectional survey of the EAPC Research Network. J Opioid Manag 2009;5:11-21.

8. Teunissen SC, Wesker W, Kruitwagen C, de Haes HC, Voest EE, de Graeff A. Symptom prevalence in patients with incurable cancer: A systematic review. J Pain Symptom Manage 2007;34:94-104. Doi: 10.1016/j. jpainsymman.2006.10.015

9. Brooksbank M. Palliative care: Where have we come from and where are we going? Pain. 2009;144:233-5. Doi: 10.1016/j. pain.2009.06.010.

10. Cherlin EJ, Carlson MD, Herrin J, Schulman-Green D, Barry CL, McCorkle R, et al. Interdisciplinary staffing patterns: do for-profit and nonprofit hospices differ? J Palliat Med 2010;13:389-94. Doi: 10.1089/jpm.2009.0306.

11. Spencer R, Nilsson M, Wright A, Pirl W, Prigerson $H$. Anxiety disorders in advanced cancer patients: Correlates and predictors of end-of-life outcomes. Cancer 2010;116:1810-9. Doi: 10.1002/ cncr.24954.

12. Mitchell AJ, Chan $M$, Bhatti $H$, Halton $M$, Grassi L, Johansen C, et al. Prevalence of depression, anxiety, and adjustment disor- der in oncological, haematological, and palliative-care settings: a meta-analysis of 94 interview-based studies. Lancet Oncol 2011;12:160-74. Doi: 10.1016/S14702045(11)70002-X.

13. Delgado-Guay M, Parsons HA, Li Z, Palmer JL, Bruera E. Symptom distress in advanced cancer patients with anxiety and depression in the palliative care setting. Support Care Cancer 2009;17:573-9. Doi: 10.1007/s00520-008-0529-7.

14. Landa E, Pérez IN, Sánchez S, Covarrubias A. Ansiedad y depresión relacionadas con presencia e intensidad de síntomas físicos en pacientes con cáncer terminal. Psicol Salud 2014;24:55-63.

15. Traeger L, Greer JA, Fernandez-Robles C, Temel JS, Pirl WF. Evidence-based treatment of anxiety in patients with cancer. J Clin Oncol 2012;30:1197-205. Doi: 10.1200/JCO.2011.39.5632.

16. Candy B, Jackson KC, Jones L, Tookman A, King M. Drug therapy for symptoms associated with anxiety in adult palliative care patients. Cochrane Database Syst Rev. 2012;10:CD004596. Doi: 10.1002/14651858.CD004596.pub2.

17. Jackson KC, Lipman AG. Drug therapy for anxiety in palliative care. Cochrane Database Syst Rev 2004;1:CD004596. Doi: 10.1002/14651858.CD004596

18. Cocker KI, Bell DR, Kidmans AD. Cognitive behaviour therapy with advanced breast cancer patients: A brief report of a pilot study. Psychooncology 1994;3:233237. Doi: 10.1002/pon.2960030310

19. Anderson T, Watson M, Davidson R. The use of cognitive behavioural therapy techniques for anxiety and depression in hospice patients: A feasibility study. Palliat Med 2008;22:814-21. Doi: 10.1177/0269216308095157.

20. Greer JA, Park ER, Prigerson HG, Safren SA. Tailoring cognitive-behavioral therapy to treat anxiety comorbid with advanced cancer. J Cogn Psychother 2010;24:294313. 
21. Chambers SK, Foley E, Galt E, Ferguson M, Clutton S. Mindfulness groups for men with advanced prostate cancer: A pilot study to assess feasibility and effectiveness and the role of peer support. Support Care Cancer 2012;20:1183-92. Doi: 10.1007/ s00520-011-1195-8.

22. Wood BC, Mynors-Wallis LM. Problemsolving therapy in palliative care. Palliat Med 1997;11:49-54.

23. Edelman S, Bell DR, Kidman AD. A group cognitive behaviour therapy programme with metastatic breast cancer patients. Psychooncology 1999;8:295-305.

24. Sloman R. Relaxation and imagery for anxiety and depression control in community patients with advanced cancer. Cancer Nurs 2002;25:432-5.

25. Savard J, Simard S, Giguère I, Ivers $H$, Morin CM, Maunsell E, et al. Randomized clinical trial on cognitive therapy for depression in women with metastatic breast cancer: Psychological and immunological effects. Palliat Support Care 2006;4:219-37.

26. Lévesque M, Savard J, Simard S, Gauthier JG, Ivers H. Efficacy of cognitive therapy for depression among women with metastatic cancer: A single-case experimental study. J Behav Ther Exp Psychiatry 2004;35:287-305.

27. Moorey S, Cort E, Kapari M, Monroe B, Hansford P, Mannix K, et al. A cluster randomized controlled trial of cognitive behaviour therapy for common mental disorders in patients with advanced cancer. Psychol Med 2009;39:713-23. Doi: $10.1017 /$ S0033291708004169.

28. Chan CW, Richardson A, Richardson J. Managing symptoms in patients with advanced lung cancer during radiotherapy: Results of a psychoeducational randomized controlled trial. J Pain Symptom Manage 2011;41:347-57. Doi: 10.1016/j. jpainsymman.2010.04.024.

29. Greer JA, Traeger L, Bemis H, Solis J, Hendriksen ES, Park ER, et al. A pilot randomized controlled trial of brief cog- nitive-behavioral therapy for anxiety in patients with terminal cancer. Oncologist 2012;17:1337-45. Doi: 10.1634/theoncologist.2012-0041.

30. Boutron I, Moher D, Altman DG, Schulz KF, Ravaud P; CONSORT Group. Extending the CONSORT statement to randomized trials of nonpharmacologic treatment: explanation and elaboration. Ann Intern Med 2008;148:295-309.

31. Hasson H. Systematic evaluation of implementation fidelity of complex interventions in health and social care. Implement Sci 2010;5:67. Doi: 10.1186/1748-5908-5-67.

32. Carroll C, Patterson M, Wood S, Booth A, Rick J, Balain S. A conceptual framework for implementation fidelity. Implement Sci 2007;2:40.

33. Dobson D, Dobson K. Evidence-based practice of cognitive behavioral therapy. New York: Guilford Publication, 2009.

34. Moorey S, Greer S. Oxford guide to CBT for people with cancer (2nd ed.). New York: Oxford University Press, 2012.

35. Nield MA, Soo Hoo GW, Roper JM, Santiago S. Efficacy of pursed-lips breathing: A breathing pattern retraining strategy for dyspnea reduction. J Cardiopulm Rehabil Prev 2007;27:237-44.

36. D'Zurilla TJ, Goldfried MR. Problem solving and behavior modification. J Abnorm Psychol. 1971;78:107-26.

37. Marrero RJ, Carballeira M. Terapia de solución de problemas en enfermos de cáncer. International J Psychol Psychol Ther 2002;2:23-39.

38. Rangel-Domínguez NE, Ascencio-Huertas L, Ornelas-Mejorada RR, Allende-Pérez SR, Landa-Ramírez E, Sánchez-Sosa JJ. Efectos de la solución de problemas sobre los comportamientos de autocuidado de cuidadores de pacientes oncológicos en fase paliativa: un estudio piloto. Psicooncología 2013;10:365-76. Doi: 10.5209/ rev_PSIC.2013.v10.n2-3.43455

39. Hayes SC, Strosahl KD, Wilson KG. Acceptance and commitment therapy: The 
process and practice of mindful change. New York:The Guilford Press. 2012.

40. Greer JA, Graham JS, Safren SA. Resolving treatment complications associated with comorbid medical conditions. In: Otto M, Hofmann S, editors. Avoiding treatment failure in anxiety disorders. New York: Springer, 2009. 9.317-46.

41. Cluver JS, Schuyler D, Frueh BC, Brescia F, Arana GW. Remote psychotherapy for terminally ill cancer patients. J Telemed Telecare 2005;11:157-9.

42. Kornblith AB, Dowell JM, Herndon JE 2nd, Engelman BJ, Bauer-Wu S, Small EJ, et al. Telephone monitoring of distress in patients aged 65 years or older with advanced stage cancer: A cancer and leukemia group B study. Cancer 2006;107:2706-14.

43. Sackett DL, Rosenberg WM, Gray JA, Haynes RB, Richardson WS. Evidence based medicine: What it is and what it isn't. BMJ 1996;312:71-2. Doi: 10.1136/ bmj.312.7023.71

44. Glasziou P, Del Mar C, Salisbury J. Evidencebased medicine workbook: Finding and applying the best research evidence to improve patient care. London: BMJ Books, 2003.

45. Parzsolt F, Ochletz A, Thim A, Gardner D. Evidence-based decision making-the six step approach. Evid Based Med 2003;8:1656. Doi.org/10.1136/ebm.8.6.165.

46. Haynes RB, Devereaux PJ, Guyatt GH. Physicians' and patients' choices in evidence based practice. BMJ 2002;324:1350. Doi: 10.1136/bmj.324.7350.1350

47. Landa E, Martínez E, Sánchez J. Medicina basada en la evidencia y su importancia en la medicina conductual. Psicol Salud 2013;23:273-82.

48. Heneghan C, Perera R, Ward AA, Fitzmaurice $D$, Meats $E$, Glasziou P. Assessing differential attrition in clinical trials: Selfmonitoring of oral anticoagulation and type II diabetes. BMC Med Res Methodol 2007;7:18. Doi:10.1186/1471-2288-7-18

49. Kazdin AE. Single-case research designs: Methods for clinical and applied settings, 2nd Edition. New York: Oxford University Press, 2011. 